\title{
Types of anti-hypertensive medication for post-transplant hypertension and risk of graft failure
}

\author{
Sehoon Park' , Sung Jin Kang ${ }^{2}$, Ji Eun Kim³ ${ }^{3}$ Yaerim Kim ${ }^{4}$, Kwangsoo Kim ${ }^{5}$, Minsu Park ${ }^{6}$, Yon Su Kim \\ Yaeji Lee ${ }^{2}$, Hajeong Lee ${ }^{7}$ \\ ${ }^{1}$ Division of Nephrology, Department of Internal Medicine, Korean Armed Forces Capital Hospital, Seongnam, Korea \\ ${ }^{2}$ Department of Applied Statistics, Chung-Ang University, Seoul, Korea \\ ${ }^{3}$ Division of Nephrology, Department of Internal Medicine, Hanyang University Guri Hospital, Guri, Korea \\ ${ }^{4}$ Division of Nephrology, Department of Internal Medicine, Keimyung University School of Medicine, Daegu, Korea \\ ${ }^{5}$ Transdisciplinary Department of Medicine \& Advanced Technology, Seoul National University Hospital, Seoul, Korea \\ ${ }^{6}$ Department of Statistics, Keimyung University, Daegu, Korea \\ ${ }^{7}$ Division of Nephrology, Department of Internal Medicine, Seoul National University Hospital, Seoul, Korea
}

Background: Additional study is warranted to reveal an appropriate anti-hypertensive medication treatment strategy for post-transplant hypertension in kidney transplant recipients.

Methods: The study included transplant recipients who maintained functioning graft for at least 1 year from transplantation, collected from the nationwide claims database of South Korea during 2008 to 2017. The usage of antihypertensive medications between 6 months to 1 year was the main exposure, and those who had inconsistent/transient usage of antihypertensive drugs were excluded. The prognostic outcome was death-censored graft failure and we also investigated the post-transplant major adverse cardiovascular events (MACEs) outcome.

Results: We included 8,014 patients without post-transplant hypertension and 6,114 recipients who received treatments for hypertension in the post-transplant period. Among them, 1,972 patients (33\%) received multiple anti-hypertensive medications, and among single-agent users, 1,690 patients (28\%) received dihydropyridine-calcium-channel-blocker, 1,807 (30\%) received beta-blockers, 475 (29\%) received renin-angiotensin-aldosterone-blockers, and $170(<1 \%)$ received other types of antihypertensive medications, respectively. Those with post-transplant hypertension had significantly worse risk of death-censored graft failure than those without (hazard ratio [HR], 1.25; 95\% confidence interval [Cl], 1.08-1.46) than those without. In addition, those who received multiple drugs showed significantly higher MACEs risk than those who did not require anti-hypertensive treatments (HR, 1.35; 95\% Cl, 1.01-1.81). Among the single-agent user, those who received beta-blocker showed significantly higher risk of death-censored graft failure ( $\mathrm{HR}, 1.39 ; 95 \% \mathrm{Cl}, 1.12-1.72)$, while those who received calcium-channel blocker showed significantly lower risks ( $\mathrm{HR}, 0.81 ; 95 \% \mathrm{Cl}, 0.65-1.00)$ than the others. The single-agent user who received renin-angiotensin-aldosterone-blockades showed similar graft prognosis with the other medication users ( $\mathrm{HR}, 0.77 ; 95 \% \mathrm{Cl}, 0.52-1.14)$. Among the multiagent users, no significant prognostic differences were identified according to the types of anti-hypertensive medications.

Conclusions: Post-transplant hypertension is associated with poor post-kidney transplant prognosis, particularly when multiple types of medications were required for treatment. This study supports that dihydropyridine calcium-channel-blocker may be considered as the initial choice for post-transplant hypertension.

Corresponding author: Sehoon Park

E-mail: mailofsehoon@gmail.com 